\title{
Primary care networks: the risk of "mission creep" calls for focused ambition
}

This letter (BMJ 2019;367:15978, doi:10.1136/bmj.15978) was missing a disclaimer when it was originally published on 15 October 2019. This has now been added online: "The views expressed are those of the authors and not necessarily those of the NHS, the NIHR, or the Department of Health and Social Care." 